\title{
Right heart thrombus - "clot-in-transit"
}

\author{
Husam M Salah ${ }^{1}$, Fuad J Habash ${ }^{2}$ and Angel Lopez Candales ${ }^{2 *}$ \\ ${ }^{1}$ Department of Internal Medicine, University of Arkansas for Medical Sciences, USA \\ ${ }^{2}$ Department of Cardiovascular Medicine, University of Arkansas for Medical Sciences, USA
}

\begin{abstract}
Introduction: Identification of a right heart $(\mathrm{RH})$ "clot-in-transit" not only is an unusual but also, a potentially deadly imaging finding.

Case presentation: We present a case of a 42-year-old Caucasian male who presented with a 3-month history of shortness of breath, tachycardia, and tachypnea. On presentation, physical examination was consistent with a volume overload status. A bedside echocardiogram showed a dilated left ventricle with a severely reduced systolic and mildly dilated right ventricle with a markedly reduced systolic function. Most importantly, a bi-lobulated, echogenic mass in the right ventricle, suggestive of a thrombus was clearly identified. A CT pulmonary angiogram was then obtained showing evidence of pulmonary embolus (PE). Patient was started on heparin
\end{abstract} drip for anticoagulation.

Discussion: A RH thrombus is associated with a very high mortality rate. Treatment options include anticoagulation with heparin, thrombolytic therapy, and surgical intervention; however, there are no clear guidelines for the management. PE was initially not suspected given a clinically picture suggestive of congestive heart failure exacerbation. This case report demonstrates the value of point-of-care bedside echocardiography in such situations, especially when official complete echocardiograms are not available. It also demonstrates that adequate training in performing these studies and having a basic of acute echocardiographic emergencies are crucial for its benefit in the acute clinical setting.

Conclusion: The use of bedside echocardiography is helpful and can further improve clinical decision making in the acute medical and emergency departments.

\section{Introduction}

A right heart $(\mathrm{RH})$ thrombus is an unusual finding most commonly first diagnosed using echocardiography. This rare clinical entity is for the most part encountered in the setting of deep venous thrombosis as a presage of pulmonary embolism (PE) or in the setting of recurrent embolization. Regardless, it is associated with an inordinate morbidity and mortality rate. The latter reported in $28 \%$ in treated patients and $80-100 \%$ in untreated patients [1].

\section{Case Presentation}

We present a case of a 42 -year-old Caucasian male with a past medical history significant for tobacco and drug use (amphetamine and marijuana) disorders who presents to the emergency department with a 3-month history of shortness of breath and acute worsening of his dyspnea 2 days prior to his presentation. Patient denied chest pain, palpitations, cough, fever, or recent weight changes. Physical examination was remarkable for a heart rate of 118 beats per minute, respiratory rate of 23 breaths per minute, and presence of significant bilateral lower extremity edema was noted. Complete blood count, basic metabolic panel, and troponin level were normal. B-type natriuretic peptide was elevated at $1230 \mathrm{pg} / \mathrm{mL}$ (normal value is $<=100 \mathrm{pg} / \mathrm{mL}$ ). Electrocardiogram (ECG) showed sinus tachycardia without significant ST segment changes.

A point-of-care bedside transthoracic echocardiogram (TTE) performed by the emergency staff showed a dilated left ventricle (LV) with a severely reduced systolic function. The estimated ejection fraction was between 15 and $20 \%$. In addition, a mildly dilated right ventricle (RV) with a markedly reduced systolic function was also identified. Emergency physicians contacted cardiology staff for admission to treat patient for heart failure. Cardiology staff reviewed the non-contrasted bedside echo images obtained by emergency physicians and noted the unrecognized presence of a RV mass.

Specifically, this mass was described as bi-lobulated, echogenic mass with central lucency and mild mobility, measuring $(1.3 \times 1.4 \mathrm{~cm}$ in the first lobe and $0.7 \times 0.7 \mathrm{~cm}$ in the second lobe). This mass located at the RV apex and adjacent to a dyskinetic wall was highly suggestive of a thrombus (Figure $1 \mathrm{~A}$ and $1 \mathrm{~B}$ ). In view of this finding, a CT pulmonary angiogram was then obtained (Figure 2) already showing evidence of PE in the left pulmonary artery with pulmonary artery dilation. Immediate anticoagulation with intravenous heparin was initiated as well as optimization of his heart failure management with diuretics, beta-blockers and ACE inhibitors. Contrast enhanced image for the $\mathrm{RV}$ thrombus was obtained the next morning as shown in Figure 1C.

\section{Discussion}

A RH thrombus is a rare finding that is usually first diagnosed using echocardiography. It is estimated to account for $4 \%$ of PE cases and is associated with a very high mortality rate $(28 \%$ in treated patients and $80-100 \%$ in untreated patients). A RH thrombus is invariable associated with a PE ( $98 \%$ of the cases and typically result from embolization that arises from thrombosis originating from the deep venous system or less common from intracardiac sources such as central lines, pacemakers, and right-sided prosthetic valves, endocarditis and atypical myxomas [1,2].

${ }^{*}$ Correspondence to: Candales AL, MD, University of Arkansas for Medical Sciences, Department of Internal Medicine, Divisions of Cardiology, $4301 \mathrm{~W}$. Markham St., \#532, Little Rock, AR 72205, USA, Tel: 501-686-7882; Fax: 501686-6439; E-mail: FHabash@uams.edu

Received: December 10, 2020; Accepted: March 18, 2021; Published: March 21, 2021 


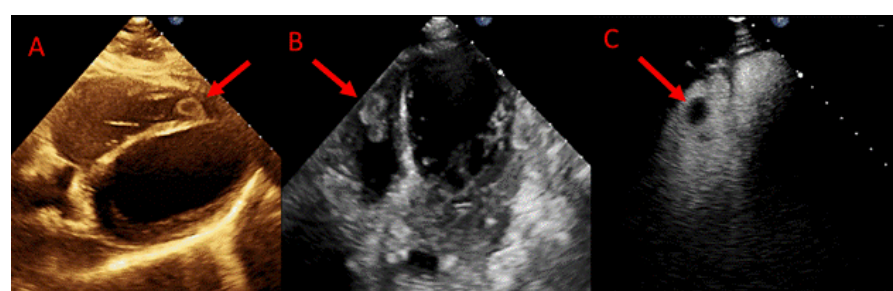

Figure 1. Echocardiographic images of RV thrombus in A (subcostal view), B (off-axis 4 chamber view) and $\mathrm{C}$ (off-axis 4 chamber view with contrast)

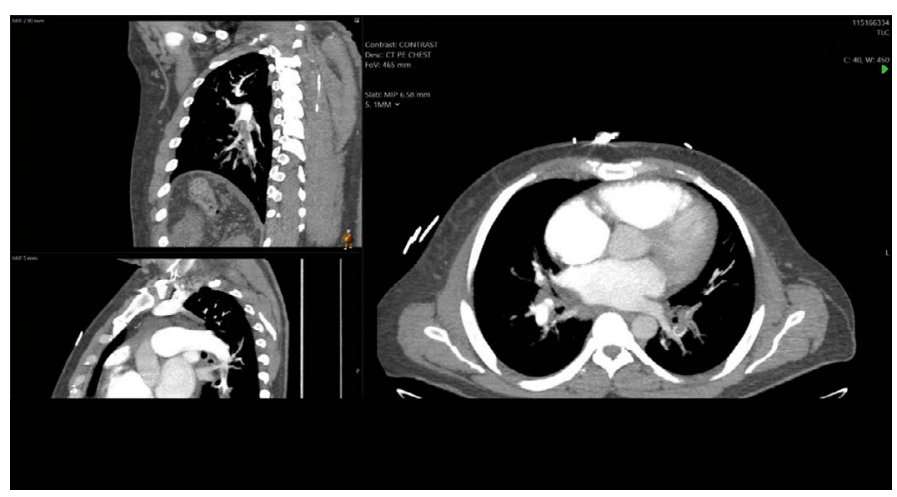

Figure 2. CT pulmonary angiogram showing a pulmonary embolus in the left pulmonary artery with pulmonary artery dilation

These RH thrombi are mainly classified according to their etiologies and morphologies. Type A thrombi are worm-like, highly mobile, and are typically associated with deep vein thrombosis. Type B thrombi have broad-based attachment to the ventricular wall, are non-mobile, and typically form in-situ due to underlying cardiac abnormalities (e.g. atrial fibrillation, cardiomyopathy, catheters). Type $\mathrm{C}$ is extremely rare and highly mobile with an appearance that resembles cardiac myxomas $[3,4]$.

Treatment options include anticoagulation with heparin, thrombolytic therapy, or thrombectomy [2] Rose, et al. performed a retrospective analysis of all reported cases (177 cases) of right heart thromboembolism between 1966 and 2000 and found better outcome with thrombolytic therapy, which was associated with an $11.3 \%$ mortality rate compared to a $28.6 \%$ mortality rate with anticoagulation therapy and a $23.8 \%$ mortality rate with surgical intervention. In our particular case, since the presentation was subacute on chronic rather than acute; and the $\mathrm{RH}$ thrombus was in effect an incidental finding; hence, a conservative approach was indicated. From a hemodynamic perspective the chronic decompensated heart failure was the likely culprit not only limiting the patient's mobility and promoting the development of deep vein thrombosis but also favoring a low-flow state that compounded thrombus formation and eventual PE.

This case also illustrates that the full benefit of point-of-care echocardiography not only is attained when adequate images are acquired but most importantly when these images are interpreted with a basic understanding of clinical imaging, particularly in the context of expected key echocardiographic findings during emergency clinical situations.

\section{Conclusions}

Presence of a RH thrombus is almost always associated with PE. Although there are no clear guidelines for the management, current options include anticoagulation with heparin, thrombolytic therapy, or surgical intervention. Use of point-of-care echocardiography is helpful and can further improve clinical decision making in the acute medical and emergency departments.

\section{References}

1. Otoupalova E, Dalal B, Renard B (2017) Right heart thrombus in transit: a series of two cases. Crit Ultrasound J 9: 14. [Crossref]

2. Rose PS, Punjabi NM, Pearse DB (2002) Treatment of right heart thromboemboli. Chest 121: 806-814. [Crossref]

3. Naeem K (2015) Floating thrombus in the right heart associated with pulmonary embolism: The role of echocardiography. Pak J Med Sci 31:233-235. [Crossref]

4. Finlayson GN (2008) Right heart thrombi: consider the cause. Can J Cardiol 24: 888. [Crossref]

Copyright: (C2021 Salah HM. This is an open-access article distributed under the terms of the Creative Commons Attribution License, which permits unrestricted use, distribution, and reproduction in any medium, provided the original author and source are credited. 